\title{
A micromechanics-based interface mesomodel for virtual testing of laminated composites
}

\author{
Pierre Ladevèze*, Federica Daghia, Emmanuelle Abisset and Camille Le Mauff
}

\author{
*Correspondence: \\ ladeveze@lmt.ens-cachan.fr \\ LMT-Cachan (ENS Cachan, \\ CNRS-UMR8535, UPMC, PRES \\ UniverSud Paris), 61 av. du Président \\ Wilson, 94235 Cachan CEDEX, \\ France
}

\begin{abstract}
Background: The prediction of the behavior of laminated composite structures up to final fracture continues to be a challenge today. Indeed, failure may occur due to the interaction of small-scale degradations, such as transverse intraply cracks and interface delamination, which are difficult to account for in calculations on the structure's scale.

Methods: Here, in order to model the interaction of intralaminar and interlaminar degradations, we develop a new and relatively simple micromechanics-based interface mesomodel which differs from classical cohesive interface models, since it includes the coupling between transverse intraply cracks and interface delamination.

Results: The new interface model was implemented in a finite element code and used in the simulation of tensile tests on unnotched and holed specimens. Simulations with a classical cohesive interface model (not including coupling) were also carried out.

Conclusions: The simulations highlight the need for introducing intra-/interlaminar's behavior coupling in order to accurately predict the damage evolution and failure stress and mode.
\end{abstract}

\section{Background}

The last quarter-century has witnessed considerable research efforts in the mechanics of composites in order to understand and predict the behavior of these materials, the ultimate goal being the design of the materials/structures/manufacturing processes. Even in the case of laminated composites, the prediction of the evolution of damage up to and including final fracture remains a major challenge which is at the heart of today's 'virtual structural testing' revolution engaged in by the aeronautical industry. Virtual structural testing consists, whenever possible, in replacing the numerous experimental tests used today by virtual tests.

An answer to the virtual structural testing challenge is what is called the 'damage mesomodel for laminated composites', developed at LMT-Cachan since the 1980s [1,2]. The main assumption is that the behavior of any laminate under any loading up to final fracture can be described using two elementary entities: the ply and the interface. The ply is described as a full three-dimensional orthotropic and damageable continuum. In particular, transverse macrocracks running parallel to the fibers (such as splits) are modeled as completely damaged zones; these may appear thicker numerically than the cracks observed experimentally. The interface is a surface entity, i.e. a cohesive interface [3].

(c) 2013 Ladevèze et al.; licensee Springer. This is an Open Access article distributed under the terms of the Creative Commons Attribution License (http://creativecommons.org/licenses/by/2.0), which permits unrestricted use, distribution, and reproduction in any medium, provided the original work is properly cited. 
An enhanced ply model based on micromechanics has been introduced in [4,5]. Today, several similar mesoscopic approaches are being developed [6].

The starting point of this paper was the need to improve the predictions of the standard mesomodel in terms of delamination. Even though it led to realistic calculated responses for complex engineering problems [7-10], it was shown to underpredict the delaminated areas in some industrially significant test cases, such as low-velocity impact [10]. This means that a standard cohesive interface model, even combined with a ply mesomodel, may not be capable of producing realistic responses in terms of delamination. A heuristic remedy was proposed in [10] and more elaborate corrections were introduced in [11,12].

The description of the interaction between delamination and transverse microcracking is a rather ancient question in micromechanics [13-22]. In all the referred works, two-dimensional discrete models are used. Both transverse intraply cracks and delamination cracks are described in detail; thus, the competition between the two mechanisms can be modeled directly. Indeed, the physics of the problem is very well-known (see the review papers [1,21-23]). Today, the difficulty lies elsewhere, namely in the fact that the discrete modeling of every single discontinuity becomes unfeasible for complex engineering problems involving several thousands of cracks. On the one hand, even with high-performance computational tools [24], the computational micromechanical model introduced in $[1,23,25]$ still leads to prohibitive computational efforts and, thus, is far from meeting the virtual structural testing requirements. On the other hand, when a mesoscale damage approach is used, some of the information regarding the detailed microscopic stress/strain state is lost. Therefore, the ply/interface coupling proposed in this article is necessary in order to restore the correct physical description in terms of transverse microcracking-induced delamination.

Apart from purely microscopic and mesoscopic approaches, intermediate approaches have recently been proposed in the literature in order to account for the interaction between transverse cracking and delamination. For example, in works such as [26,27], classical cohesive interfaces are used for both transverse cracks and delaminations; in this case, however, a priori information about the cracking pattern (e.g. the position of the splits) needs to be introduced in order to carry out the simulations. Another approach consists in introducing discrete cracks thanks to techniques such as the $\mathrm{X}$-FEM [28]; once again, the interaction between transverse cracks and delamination occurs naturally, but the local stress/strain field is still poorly represented compared to a purely microscopic approach, and a minimum crack spacing (which is generally much larger than in reality) related to the element size chosen needs to be introduced. These intermediate approaches are helpful for one's understanding of the degradation mechanisms. Unfortunately, because of the approximations introduced in the physics and the $a$ priori information which they require, they cannot be considered to be predictive models.

In this paper, we present a new and relatively simple micromechanics-based interface model which takes into account the interaction between delamination and microcracking. We consider an $(\alpha /-\alpha)$ interface between two plies with different microcracking densities; both in-plane and out-of-plane mesostresses are taken into account. In the first Section, the classical micromechanical description of the damage mechanisms and the main features of the bridge between micro- and mesomechanics $[4,5,29]$ are reviewed. Out-of-plane mesostresses are discussed in the second Section, in which the homogenized interface stiffness is derived using what is known as the basic interface problem, 
which is part of the micro-meso bridge [29]. This problem, defined over a 3D cell, is solved numerically for realistic situations involving out-of-plane mesostresses. Classical interface damage evolution laws are retained because their identification relies on standard delamination tests. In-plane mesostresses are discussed in the third Section using, once again, the basic interface problem. In-plane mesostresses can induce local delamination at the tips of transverse microcracks after saturation of the microcracking mechanism. It is shown that these local delaminations are generally unstable and, therefore, a criterion for the delamination of an interface, associated to the mesostress state of each adjacent ply, is proposed. In order to illustrate the predictive capabilities of the enhanced interface mesomodel and the importance to introduce it to ensure sufficient predictive capabilities to the model, we use the example of a simple tensile test, namely the $\left[0_{m} / 90_{n}\right]_{s}$, and a more structural one namely an open-hole tensile test (fourth Section). No further information concerning the cracking pattern is introduced in the model.

\section{Methods}

\section{The damage mechanisms on the microscale}

Four scenarios can be distinguished. The first two mechanisms have been studied for laminated composites by the micromechanics community (see the reviews [1,21-23]). Matrix microcracking (Scenario 1) is driven by the ply's microstructure: usually, matrix microcracks originate perpendicular to the plane of the ply, then run throughout the ply's thickness, and finally grow parallel to the fibers' direction. Moreover, the microcracking pattern can be considered to be locally periodic: thus the amount of microcracking can be quantified by the microcracking rate $\rho=H / L$, where $H$ is the ply thickness and $L$ the distance between two cracks (see Figure 1). Local delamination (Scenario 2) generally occurs after the saturation of matrix microcracking: it is caused by the stress concentrations at the tips of the intraply cracks. This mechanism is quantified by the local delamination ratio $\tau=e / H$, where $e$ is the length of the delaminated zone (see Figure 1). Diffuse intra- and interply damage mechanisms (Scenarios 3 and 4) were introduced into the damage mechanics of laminates a long time ago, but they are usually not taken into

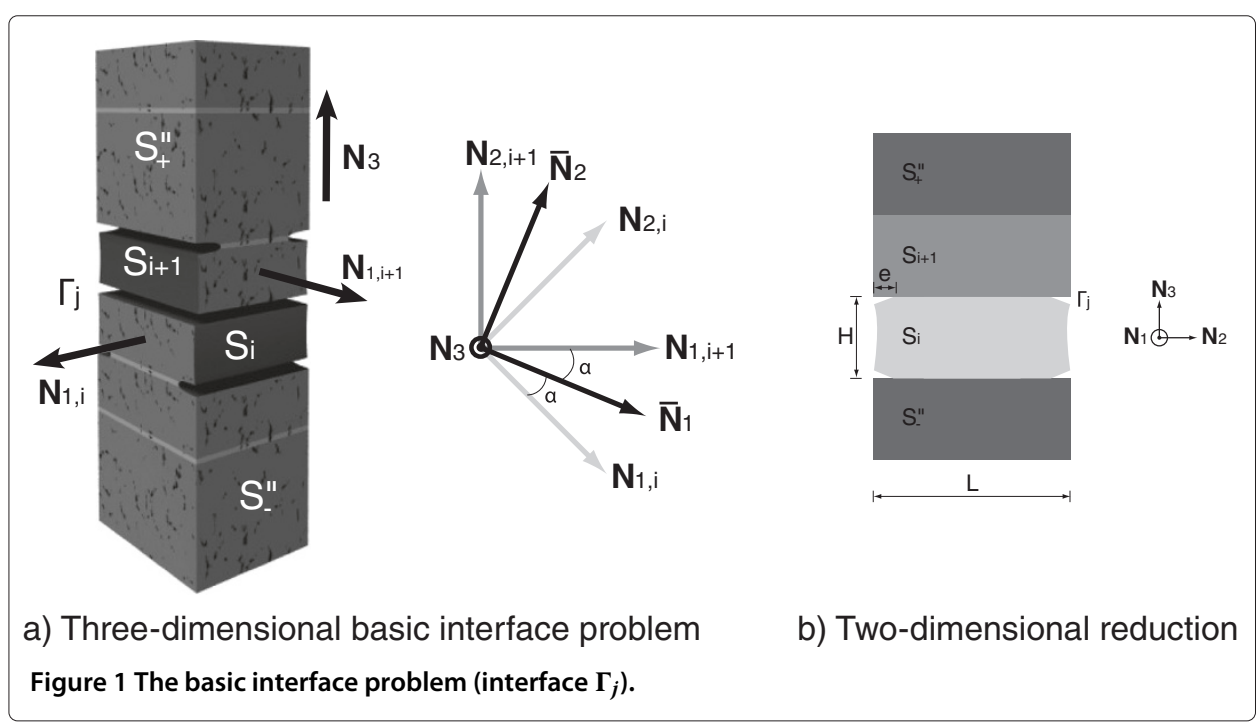


account in micromechanics. As they occur at the fiber's scale, they can be homogenized and they are introduced directly through damage variables and evolution laws at the ply's scale.

In order to handle these mechanisms, a computational micromodel was introduced in $[1,23]$ and developed in $[24,29]$. This micromodel reproduces the key points observed in the micromechanics of laminates $[1,23]$ quite well.

\section{The bridge between micromechanics and mesomechanics}

The enhanced damage mesomodel $[5,11,12]$ is the homogenized version of the micromechanical model introduced in [1]. The details of the bridge we derived between micromechanics and mesomechanics are given in $[4,5,29]$. The idea is to impose that the potential energy stored in the plies and in the interfaces must be the same on the microscale and on the mesoscale, which leads to the following relation between the microquantities and mesoquantities:

$$
\pi \varepsilon_{m e s o} \pi=\frac{1}{\operatorname{mes}(\Gamma)} \int_{\Gamma} \pi \varepsilon_{\text {micro }} \pi \mathrm{d} S, \quad \sigma_{m e s o} \mathbf{N}_{3}=\frac{1}{\operatorname{mes}(\Gamma)} \int_{\Gamma} \sigma_{m i c r o} \mathbf{N}_{3} \mathrm{~d} S
$$

where $\pi$ is the projection operator onto the plane and $\Gamma$ is an arbitrary section of the unit cell perpendicular to vector $\mathbf{N}_{3}$ (see Figure 1). Thus, there are two basic problems, one associated with in-plane loading and the other associated with out-of-plane loading.

The problem associated with out-of-plane loading, which defines the mesodescription of the interface, is summarized in Figure 1. Considering an interface $\Gamma_{j}$ (in this case, a 3D matrix layer of thickness $\frac{H_{e}}{20}$, where $H_{e}$ is the thickness of the elementary ply) between two cracked plies $S_{i}$ and $S_{i+1}$, the upper part $S_{+}^{\prime \prime}$ and the lower part $S_{-}^{\prime \prime}$ of the laminate are homogenized. Periodic boundary conditions are defined. Uniform elementary loadings are introduced on the cracked surfaces: this residual problem can be superposed to an uncracked problem in order to obtain the full solution of the cracked cell under elementary loadings. More details on the definition of the interface problem can be found in [29].

Using the finite element method, the 3D reference problem on the microscale was solved for different sets of parameters (thickness, stiffness, $\rho \in[0,0.7], \tau \in[0,0.4]$ ) which are likely to be encountered in practice, leading to a set of mesodamage indicators associated to the preferential directions of the interface $\left(\overline{\mathbf{N}}_{1}, \overline{\mathbf{N}}_{2}\right)$ defined in Figure 1. It was shown that the mesodamage of the interface depends only on the interface itself and on the microcracking rates of the adjacent plies [29].

\section{The interface's damage mesomodel - the concept of interface stiffness}

First, let us study the change in the stiffness of the interface mesomodel due to microcracking in the adjacent plies in the general case of different microcracking rates. To obtain these stiffness changes, the basic interface problem must be solved under out-of-plane loading. With only a limited loss of accuracy, one can consider the solution to be the superposition of the solutions of two 2D problems (one of which is depicted in Figure 1), which are associated with the fiber directions of Ply $S_{i}$ and Ply $S_{i+1}$ [29].

\section{Properties of the basic 2D interface problem}

The basic $2 \mathrm{D}$ interface problem is defined in Figure 1 . Since the results are quasiindependent of the stacking sequence, a sequence of [90/0/902] with $x \equiv \mathbf{N}_{1}$ was chosen. 
$h$ denotes the interface's thickness; the main parameters are the microcracking rate $\rho$ and the delamination ratio $\tau$. The typical properties of carbon/epoxy unidirectional plies are considered:

$$
\begin{aligned}
E_{1} & =148 \mathrm{GPa}, \quad E_{2,3}=10.8 \mathrm{GPa}, \quad v_{12,13}=0.3, \quad \nu_{23}=0.4, \\
G_{12,13} & =5.8 \mathrm{GPa}, \quad G_{23}=\frac{E_{2}}{2\left(1+\nu_{23}\right)}, H=H_{e}=0.125 \cdot 10^{-3} \mathrm{~m} .
\end{aligned}
$$

For the interfaces, which are considered to be thin 3D matrix layers made of isotropic material, the material properties are: $E=2.4 \mathrm{GPa}, \quad v=0.33, h=H_{e} / 20$.

The problem to be solved is elastic and follows the generalized plane strain assumption (i.e. the displacement in direction $\mathbf{N}_{1}$ is constant). It has been proven that the mesobehavior of interface $\Gamma_{j}$ depends only on interface $\Gamma_{j}$ and ply $S_{i}$, i.e. on parameters $\lambda=2 \tau \rho$, $\rho$ and on the ply thickness [29].

The cell was analyzed for unit values of stresses $\sigma_{33}, \sigma_{23}, \sigma_{13}, \sigma_{22}$ and $\sigma_{12}$ using a relatively refined $\mathrm{FE}$ mesh, leading to a residual energy expressed as a surface energy:

$$
\begin{aligned}
\Delta e= & c_{33}\left(\sigma_{33}\right)^{2}+c_{23}\left(\sigma_{23}\right)^{2}+c_{13}\left(\sigma_{13}\right)^{2}+c_{22}\left(\sigma_{22}\right)^{2}+c_{12}\left(\sigma_{12}\right)^{2} \\
& +c_{3313} \sigma_{33} \sigma_{13}+c_{3323} \sigma_{33} \sigma_{23}+c_{1323} \sigma_{13} \sigma_{23}+c_{2212} \sigma_{22} \sigma_{12}+c_{2233} \sigma_{22} \sigma_{33} \\
& +c_{2213} \sigma_{22} \sigma_{13}+c_{2223} \sigma_{22} \sigma_{23}+c_{1233} \sigma_{12} \sigma_{33}+c_{1213} \sigma_{12} \sigma_{13}+c_{1223} \sigma_{12} \sigma_{23}
\end{aligned}
$$

The values of the coupling coefficients have been computed

$$
\alpha_{i j k l}=\frac{c_{i j k l}}{\left(c_{i j} c_{k l}\right)^{\frac{1}{2}}}, \quad \bar{\alpha}_{i j k l}=\left.\max \right|_{\text {calculated points }}\left|\alpha_{i j k l}\right|
$$

and the calculated points were $\tau=(0.1,0.2)$ and $\rho=(0.2,0.4,0.6,0.8)$.

Except for $c_{2233}$, these coupling coefficients are negligible, the maximum being around $6.1 \cdot 10^{-13}$. Thus, $\Delta e$ can be taken as:

$$
\Delta e=c_{33}\left(\sigma_{33}\right)^{2}+c_{23}\left(\sigma_{23}\right)^{2}+c_{13}\left(\sigma_{13}\right)^{2}+c_{22}\left(\sigma_{22}\right)^{2}+c_{12}\left(\sigma_{12}\right)^{2}+c_{2233} \sigma_{22} \sigma_{33}
$$

Moreover, the last three terms, which are proportional to $h$, are small compared to the ply's residual energy, which is proportional to $H$, so they, too, are negligible. Consequently, the interface's residual energy can be taken as:

$$
\Delta e=c_{33}\left(\sigma_{33}\right)^{2}+c_{23}\left(\sigma_{23}\right)^{2}+c_{13}\left(\sigma_{13}\right)^{2}
$$

Now, let us introduce approximations for coefficients $c_{33}, c_{13}$ and $c_{23}$, which depend on $\lambda=2 \tau \rho$ and $\rho$. These approximations are derived from the analysis of the extreme cases: small $\rho$, large $\rho$ and $\lambda$ equal to 0 or 1 .

Let us introduce the damage parameters $d_{33, i}, d_{13, i}$ and $d_{23, i}$ associated to the 2D basic interface problem involving Ply $S_{i}$ :

$$
\frac{d_{33, i}}{1-d_{33, i}}=c_{33} \frac{2 E}{h}, \quad \frac{d_{13, i}}{1-d_{13, i}}=c_{13} \frac{2 G}{h}, \quad \frac{d_{23, i}}{1-d_{23, i}}=c_{23} \frac{2 G}{h}
$$

As shown in Figure 2, the following approximations work quite well:

$$
\bar{d}_{33, i}=\lambda, \quad \bar{d}_{13, i}=\lambda, \quad \frac{\bar{d}_{23, i}}{1-\bar{d}_{23, i}}=\frac{\lambda}{1-\lambda}+A(\rho), \quad A(\rho)=\frac{a(\rho)}{1-a(\rho)}
$$

with the material function $a(\rho)$ assumed to be linear $(a(\rho)=0.5 \rho$ for the material studied). 


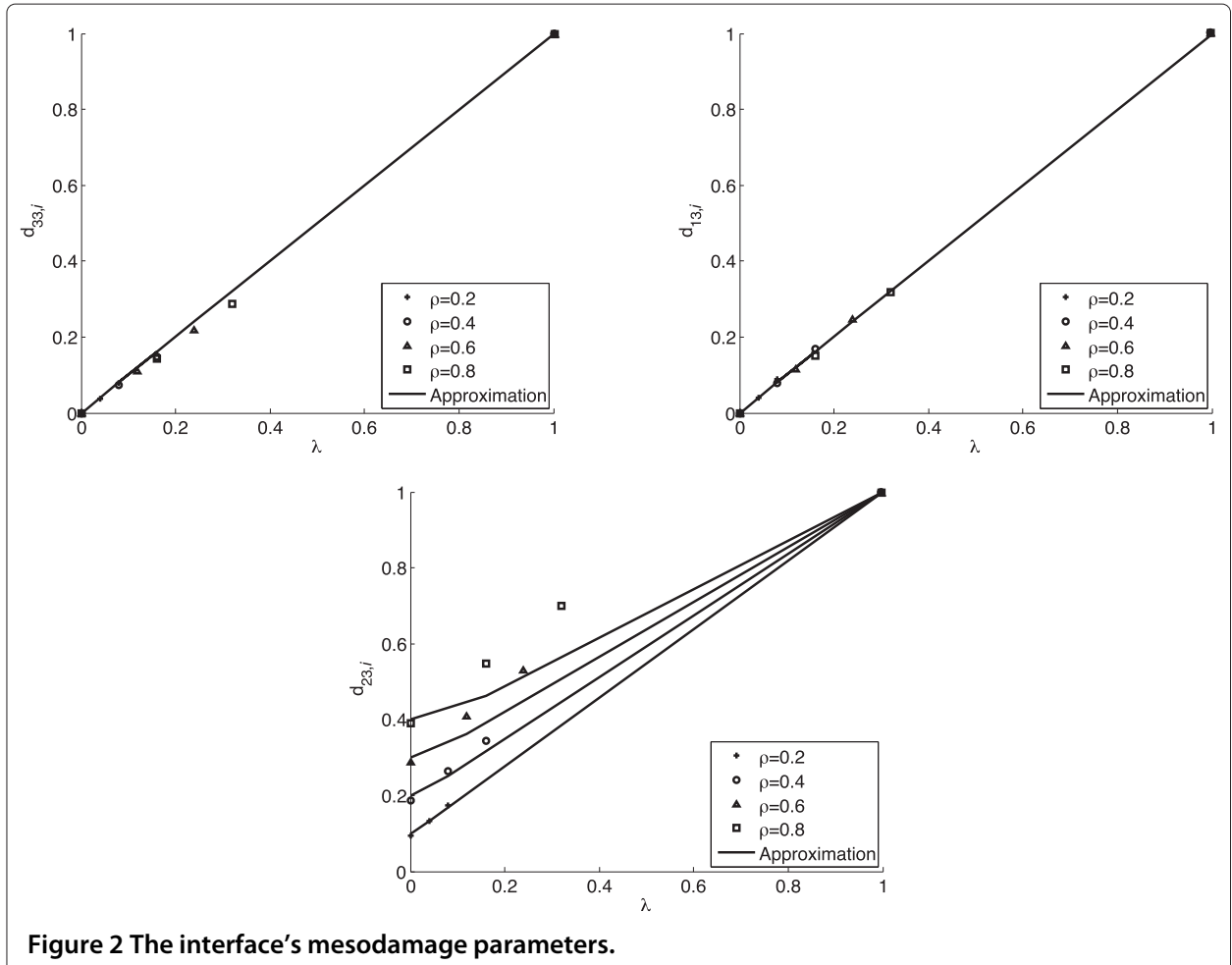

Taking into account the delaminated area in the other direction $\mathbf{N}^{\prime}{ }_{1}$, one gets:

$$
\Delta e \sim \frac{h}{2}\left[\frac{\lambda}{1-\bar{\lambda}}\left(\frac{\sigma_{13}^{2}}{G}+\frac{\sigma_{33}^{2}}{E}\right)+\frac{\sigma_{23}^{2}}{G}\left(\frac{\lambda}{1-\bar{\lambda}}+A(\rho)\right)\right]
$$

where $\lambda=2 \tau \rho=\frac{e}{L}, \lambda^{\prime}=2 \tau^{\prime} \rho^{\prime}$ and $(1-\bar{\lambda})=\left(1-\lambda^{\prime}\right)(1-\lambda)$.

\section{The microcracking/stiffness interaction of the interface mesomodel}

One must add up the residual energies of the two basic 2D interface problems:

$$
\Delta e=\frac{h}{2}\left[\frac{\lambda+\lambda^{\prime}}{1-\bar{\lambda}} \frac{\sigma_{33}^{2}}{E}+\frac{\sigma_{13}^{2}+\sigma_{23}^{2}}{G} \frac{\lambda}{1-\bar{\lambda}}+\frac{\sigma_{1^{\prime} 3}^{2}+\sigma_{2^{\prime} 3}^{2}}{G} \frac{\lambda^{\prime}}{1-\bar{\lambda}}+\frac{\sigma_{23}^{2}}{G} A(\rho)+\frac{\sigma_{2^{\prime} 3}^{2}}{G} A\left(\rho^{\prime}\right)\right]
$$

Let $\bar{\sigma}_{33}, \bar{\sigma}_{13}$ and $\bar{\sigma}_{23}$ be the mesostress components written in the interface's basis $\left(\overline{\mathbf{N}}_{1}, \overline{\mathbf{N}}_{2}\right)$ and let $2 \alpha$ be the angle between the fiber directions of the adjacent plies. One has:

$$
\begin{aligned}
& \sigma_{13}=\cos \alpha \bar{\sigma}_{13}-\sin \alpha \bar{\sigma}_{23}, \quad \sigma_{23}=\sin \alpha \bar{\sigma}_{13}+\cos \alpha \bar{\sigma}_{23} \\
& \sigma_{1^{\prime} 3}=\cos \alpha \bar{\sigma}_{13}+\sin \alpha \bar{\sigma}_{23}, \quad \sigma_{2^{\prime} 3}=-\sin \alpha \bar{\sigma}_{13}+\cos \alpha \bar{\sigma}_{23}
\end{aligned}
$$

Neglecting the term $\lambda \lambda^{\prime}$ which is very small compared to 1 , one easily obtains:

$$
\begin{aligned}
\Delta e= & \frac{h}{2}\left\{\frac{\bar{\lambda}}{1-\bar{\lambda}} \frac{\bar{\sigma}_{33}^{2}}{E}+\frac{\bar{\sigma}_{13}^{2}}{G}\left[\frac{\bar{\lambda}}{1-\bar{\lambda}}+\left(A+A^{\prime}\right) \sin ^{2} \alpha\right]+\frac{\bar{\sigma}_{23}^{2}}{G}\left[\frac{\bar{\lambda}}{1-\bar{\lambda}}+\left(A+A^{\prime}\right) \cos ^{2} \alpha\right]\right. \\
& \left.+\frac{2 \bar{\sigma}_{13} \bar{\sigma}_{23} \sin \alpha \cos \alpha}{G}\left(A-A^{\prime}\right)\right\}
\end{aligned}
$$


The new interface mesomodel - stiffness and damage

Let us note that the interface mesomodel is described as a cohesive interface with a very small 'thickness' compared to the cell's dimensions. The contributions due to microcracking should be viewed as relatively long-wavelength contributions. Thus, the energy of the interface mesomodel is:

$$
\bar{e}=\frac{h}{2}\left[\frac{\left\langle-\sigma_{33}\right\rangle^{2}}{E}+\frac{\left\langle\sigma_{33}\right\rangle^{2}}{E\left(1-d_{33}\right)}+\frac{\bar{\sigma}_{13}^{2}}{G\left(1-d_{13}\right)}+\frac{\bar{\sigma}_{23}^{2}}{G\left(1-d_{23}\right)}+\frac{\omega}{G} \bar{\sigma}_{13} \bar{\sigma}_{23}\right]
$$

where the purpose of the positive part $\langle\bullet\rangle$ is to account for crack opening and crack closure. The usual damage variables, deduced from the micro-meso energy equivalence, are:

$$
d_{33}, \quad d_{13}=\frac{d_{33}+\left(1-d_{33}\right)\left(A+A^{\prime}\right) \sin ^{2} \alpha}{1+\left(1-d_{33}\right)\left(A+A^{\prime}\right) \sin ^{2} \alpha}, \quad d_{23}=\frac{d_{33}+\left(1-d_{33}\right)\left(A+A^{\prime}\right) \cos ^{2} \alpha}{1+\left(1-d_{33}\right)\left(A+A^{\prime}\right) \cos ^{2} \alpha}
$$

with the coupling term $\omega$ written as $\omega=2 \sin \alpha \cos \alpha\left(A-A^{\prime}\right)$.

In previous papers [11,12], a simplified expression was considered, based on $\bar{\rho}=\frac{\rho+\rho^{\prime}}{2}$. This expression is equivalent to (13) for $\rho=\rho^{\prime}$, $\tau$ small (i.e. $d_{33} \rightarrow 0$ ) and $\alpha \sim 45^{\circ}$.

It is remarkable that this energy depends only on $\rho, \rho^{\prime}$ and $\bar{\lambda}$. As mentioned previously, $a(\rho)$ is a material function which can be identified from the basic $2 \mathrm{D}$ interface problem. In the present work, we used a linear law.

\section{Computation of the dissipation}

The dissipation work associated with the new interface model is:

$$
\dot{D}=\Delta \dot{e}=Y_{I} \dot{d}_{33}+Y_{I I} \dot{d}_{13}+Y_{I I I} \dot{d}_{23}+\frac{h}{2 G} \dot{\omega} \bar{\sigma}_{13} \bar{\sigma}_{23}
$$

where $\dot{\omega}$ depends on $\dot{\rho}$ and $\dot{\rho}^{\prime}$.

One can easily see that $\dot{D} \geq 0$, as $\dot{d}_{33}, \dot{\rho}$ and $\dot{\rho}^{\prime}$ are positive or equal to zero. Using (11) and (7):

$$
\begin{aligned}
\dot{D}= & \frac{h}{2}\left[\frac{\dot{d}_{33}}{\left(1-d_{33}\right)^{2}}\left(\frac{\bar{\sigma}_{33}^{2}}{E}+\frac{\bar{\sigma}_{13}^{2}+\bar{\sigma}_{23}^{2}}{G}\right)+\left(\bar{\sigma}_{13} \sin \alpha+\bar{\sigma}_{23} \cos \alpha\right)^{2} \frac{\dot{A}}{G}\right. \\
& \left.+\left(\bar{\sigma}_{13} \sin \alpha-\bar{\sigma}_{23} \cos \alpha\right)^{2} \frac{\dot{A}^{\prime}}{G}\right]
\end{aligned}
$$

Since $\dot{A}=\frac{\mathrm{d} A}{\mathrm{~d} \rho} \dot{\rho}$ is positive, it follows that $\dot{D} \geq 0$; thus, the interface mesomodel is compatible with the principles of thermodynamics.

\section{The damage mesomodel - delamination criteria}

Two different fracture mechanisms should be considered for out-of-plane loading and in-plane loading. The first fracture mechanism, associated with out-of-plane loading, is described through classical interface damage laws involving the normal stress vector.

The second fracture mechanism is due to in-plane stresses leading to microdelamination cracks at the tips of the transverse microcracks in plies. This is shown to be an unstable mechanism with a characteristic length of the same order of magnitude as the cell's dimensions. 


\section{Delamination criterion for out-of-plane loading}

The standard interface model is extended as follows. The elementary damage forces are $Y_{I}, Y_{I I}$ and $Y_{I I I}$ defined as:

$$
Y_{I}=\frac{1}{2} \frac{h}{E} \frac{\left\langle\bar{\sigma}_{33}\right\rangle^{2}}{\left(1-d_{33}\right)^{2}}, \quad Y_{I I}=\frac{1}{2} \frac{h}{G} \frac{\bar{\sigma}_{13}^{2}}{\left(1-d_{13}\right)^{2}}, \quad Y_{I I I}=\frac{1}{2} \frac{h}{G} \frac{\bar{\sigma}_{23}^{2}}{\left(1-d_{23}\right)^{2}}
$$

The effective damage force, which is responsible for the increase in the interface's damage, is:

$$
Y=\left[\left(Y_{I}\right)^{r}+\left(\gamma_{I I} Y_{I I}\right)^{r}+\left(\gamma_{I I} Y_{I I I}\right)^{r}\right]^{1 / r}
$$

where $\gamma_{I I}$ and $\gamma_{I I I}$ are two equal material coupling coefficients and the exponent $r$, which is also a material constant, is generally taken as 1 . One has:

$$
d_{33}=\left(\frac{n}{n+1} \frac{\left\langle\bar{Y}-Y_{0}\right\rangle_{+}}{Y_{c}-Y_{0}}\right)^{n} \text { if } d_{33}<1, \quad d_{33}=d_{13}=d_{23}=1 \quad \text { otherwise }
$$

where $\left.\bar{Y}\right|_{t}=\left.\sup _{\tau \leq t} Y\right|_{\tau}$ and $k, n, Y_{c}, Y_{0}$ are material constants which can be identified using standard delamination tests. Let us note that the interface mesomodel is independent of the angle $2 \alpha$ between the fiber directions of the adjacent plies.

\section{Delamination criteria for in-plane loading}

In order to analyze the microdelamination due to in-plane loading, let us review the modeling of transverse microcracking going back to the basic $2 \mathrm{D}$ interface problem.

\section{The modeling of microcracking}

Using finite fracture mechanics $[14,21,30]$, the fracture criterion is classically written as:

$$
\begin{gathered}
l(\rho, \sigma) \equiv\left(\frac{G_{I}^{u}\left\langle\tilde{\sigma}_{22}\right\rangle^{2}}{G_{I}^{c}}+\frac{G_{I I}^{u} \tilde{\sigma}_{12}^{2}}{G_{I I}^{c}}+\frac{G_{I I I}^{u} \tilde{\sigma}_{23}^{2}}{G_{I I I}^{c}}\right) \eta, \\
\eta=\frac{H}{H_{e}} \text { for } \frac{H}{\bar{H}} \leq 1, \quad \eta=\frac{\bar{H}}{H_{e}} \text { for } \frac{H}{\bar{H}} \geq 1
\end{gathered}
$$

where the unit finite energy release rates $G_{I}^{u}, G_{I I}^{u}$ and $G_{I I I}^{u}$ are calculated using the 'derivative'

$$
\check{f}(\rho)=\frac{f\left(\frac{2 \rho}{q}\right)-f\left(\frac{\rho}{q}\right)}{\frac{\rho}{q}}
$$

$q$ being a parameter (equal to about 1.5) associated with the stochastic behavior of microcracking [22]. The effective stress $\tilde{\sigma}$ is considered and $\bar{H}$ is the transition thickness between thick ply and thin ply behavior.

The fracture model is relatively simple:

$$
\left\{\begin{array}{l}
\dot{\rho} \geq 0 \quad l(\rho, \tilde{\sigma}) \leq 1 \\
\dot{\rho}[l(\rho, \tilde{\sigma})-1]=0
\end{array}\right.
$$

Remark The transverse damage $d_{22}$ associated with $\tilde{\sigma}_{22}$ is a function of $\rho$ which tends to $d_{22}=1$ for large values of $\rho$.

The solving of the $2 \mathrm{D}$ generic basic interface problem leads also to a residual energy of the layer adjacent to the interface in term of out of plane stresses. However, for the ply this contribution is not as important as the contribution over the interface which explains why 
it is not introduced in the present version of the enhanced mesomodel $[5,11,12]$. However, it will be considered in a companion paper.

The part of the plies in contact with a completely delaminated interface should behave, regarding microcracking, as half a ply $[21,22]$.

\section{The modeling of microdelamination}

With $\rho$ constant, the energy release rates related to microdelamination can be calculated as $\lambda$-derivatives. For $\tau=0$, they are equal to zero. Let us use finite fracture mechanics again and consider the $\tau$ values:

$$
\tau=(0.05,0.1,0.15,0.2) ; \quad \Delta \tau=0.05
$$

The curves giving the unit energy release rates are shown in Figure 3. It follows that the initiation criterion can be defined as:

$$
g(\tilde{\sigma}, \rho)=\left[\frac{Q_{22}^{u}\left\langle\tilde{\sigma}_{22}\right\rangle^{2}}{Q^{c}}+\frac{\gamma_{12} Q_{12}^{u} \tilde{\sigma}_{12}^{2}}{Q^{c}}\right] \frac{H}{H_{e}}
$$

where $Q_{22}^{u}$ and $Q_{12}^{u}$ are the unit energy release rates associated to $\rho, \tau=0.05, \Delta \tau=0.05$. $Q^{c}$ and $\gamma_{12}$ are critical material values. One has:

$$
\left\{\begin{array}{l}
\dot{\tau} \geq 0 \quad g(\tilde{\sigma}, \rho) \leq 1 \\
\dot{\tau}[g(\tilde{\sigma}, \rho)-1]=0
\end{array}\right.
$$

Here, the out-of-plane effective stress $\tilde{\sigma}_{23}$ is not considered. Indeed, it is negligible except in high-gradient zones (e.g. because of edge effects), in which case it is taken into account by the interface model.

The curves of Figure 3 are either increasing or flat and show that in most cases the microdelamination mechanism is unstable. When it is activated, one can consider that the interface has been completely fractured; thus, Equations (23)-(24) can be viewed as a mesodelamination criterion.

\section{A remark on the identification of the mesodelamination criterion}

The criterion given in Equations (23)-(24) depends on two material constants $Q^{c}$ and $\gamma_{12}$ which can be identified by taking advantage of available experimental results related to microcracking saturation.

Let us consider the case $\tilde{\sigma}_{22} \neq 0, \tilde{\sigma}_{12}=0$. From $\left[0_{m} / 90_{n}\right]_{s}$ tensile tests, one can identify the material constant $\rho_{s}$ which represents the microcracking density at saturation [5].
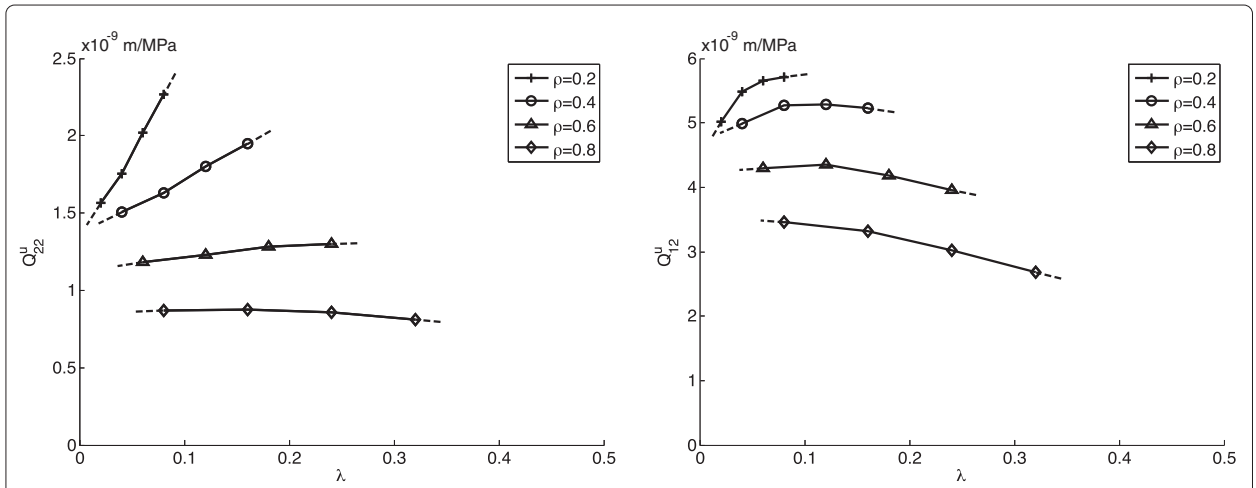

Figure 3 The unit energy release rates $Q_{22}^{u}$ and $Q_{12}^{u}$ related to microdelamination (parameter: $\rho$ ). 
From an energy point of view, this saturation is associated to the decrease in the microcracking strain energy release rate and the corresponding nearly constant strain energy release rate for microdelamination (see Figure 4). This quantity is associated with the onset of microdelamination. It follows that $Q^{c}$ can be identified as:

$$
Q^{c}=\frac{Q_{22}^{u}\left(\rho_{s}\right)}{G_{I}^{u}\left(\rho_{s}\right)} G_{I}^{c} \text { (ply) }
$$

where $Q^{u}$ and $G_{I}^{u}$ are evaluated for $\rho=\rho_{s}$. In the case $\tilde{\sigma}_{12} \neq 0, \tilde{\sigma}_{22}=0$, a saturation value seems to exist, but it may be different from that observed in mode I.

Remark The constant $\gamma_{12}$ can be identified from a tensile test of a $[+45 /-45]_{n s}$ stacking sequence or a tensile test of a holed specimen, in which shear plays an important role. Otherwise, one can take the value related to the interface model.

\section{The new interface mesomodel - in-plane loading}

The following criterion is added to the interface mesomodel:

- if $g(\tilde{\sigma}, \rho)<1$ and $g^{\prime}\left(\tilde{\sigma}^{\prime}, \rho\right)<1$, then no extra condition; otherwise, $d_{33}=1$.

$g(\tilde{\sigma}, \rho)$ and $g^{\prime}\left(\tilde{\sigma^{\prime}}, \rho^{\prime}\right)$ are associated with the adjacent plies of the interface being considered.

\section{Results and discussion}

The objective of this section is to illustrate the improvement brought by the new interface model described in this paper. One should note that this is not a complete experimental validation, but an example to demonstrate the need for the in-plane mesodelamination criterion in some classical test cases.

To do this, two different interface models are used and compared: the enhanced model described in this paper and a more classical cohesive interface model which does not include the coupling between the ply and interface behavior.

In a first time, the enhanced model is tested on a classical tension test in order to demonstrate its capability to mirror simple tests and to predict damage evolutions.

In a second time, a more complete comparison is performed with the two models, based on a structural test case: an open-hole tensile test on a quasi-isotropic laminate. This example allows then, on one hand, to highlight the need of introducing the intrainterlaminar coupling to mirror correctly the damage evolution, and, on the other hand, to
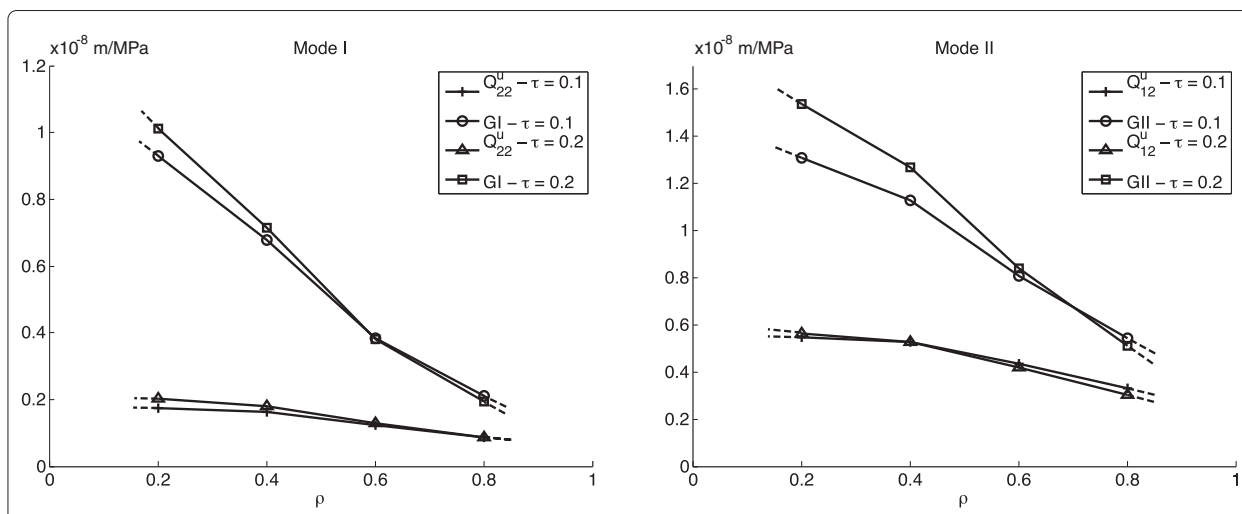

Figure 4 The unit energy release rates $G_{I}^{u}$ and $Q_{22}^{u}$ and $G_{I I}^{u}$ and $Q_{12}^{u}$ as functions of the microcracking rate $\rho$ for different values of $\tau$. 
illustrate the improvement brought by the enhanced model in the accuracy of the damage state prediction.

\section{Tension test on $\left[0 / \mathbf{9 0}_{4}\right]_{s}:$ a first validation of the proposed interface model}

Experimental results are well-known and analysed in many papers such as [21]. The top part of Figure 5 gives an overview of the sequence of damage mechanisms. Three dimensional finite element calculations are performed with a very refined mesh, a small initial defect being introduced at the center of the plate. The elastic material properties used in the simulation are the same as the ones given in Section Properties of the basic 2D interface problem. As for the parameters associated to fiber breaking and diffuse damage, they are taken as typical values for carbon/epoxy composites. The energy release rates associated with transverse cracking are: $G_{I}^{c}=200 \mathrm{~J} / \mathrm{m}^{2}$ and $G_{I I}^{c}=800 \mathrm{~J} / \mathrm{m}^{2}$. The ones related to the interfaces are assumed to be the same. Finally, the values of the parameters introduced are taken from the curves shown in the previous section. The enhanced interface model is used combined with the ply mesomodel $[5,11,12]$.

Figure 5 shows that the simulation reproduces correctly the damage physics. Until (1), transverse microcracking development is observed. Diffuse damage remains weak and is not shown in the damage charts. From (1) to (2), delamination develops very quickly and, in the end, the specimen fails by fiber failure.

For this test case, a finite element calculation carried out with a classical cohesive interface, would not reproduce correctly the interface damage physics. Indeed, in this type of model, the delamination is activated by out-of-plane stresses which are really small in these cases and would not be sufficient to activate the damage mechanism.

Moreover, the enhanced interface model proposed in this paper bring a real improvement in the damage prediction compared to the former model used previously as in [12]. Indeed, this former model uses the mean value of the microcracking densities in the two adjacent plies of the interface to trigger delamination. Then, in this particular case where only one adjacent ply of the interface is damaged, the former model fails in predicting the interface breaking.

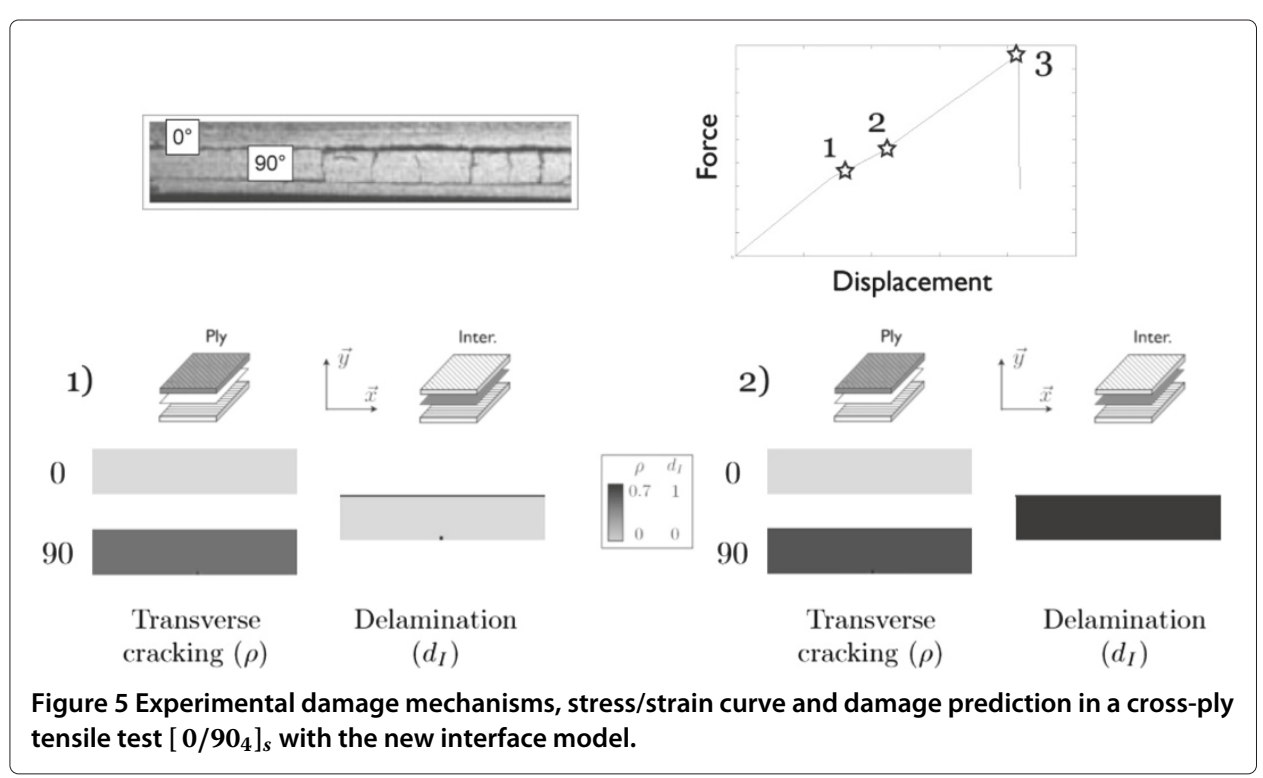




\section{Open-hole tensile test: need of the coupling introduction}

The test case used hereafter is part of Wisnom and Hallet's experimental campaign on open-hole tensile tests [31]. Series of tests were carried out on quasi-isotropic IM7/8552 carbon/epoxy specimens with a $\left[45_{m} / 90_{m} /-45_{m} / 0_{m}\right]_{n s}$ lay-up and the geometry described in Figure 6.

The lay-up of the specimen chosen for this illustration is $\left[45_{4} / 90_{4} /-45_{4} / 04\right]_{S}$ with a ply thickness $h=0.5 \mathrm{~mm}$, the hole diameter is $D=6.35 \mathrm{~mm}$ and the ratio $W / D=5$. Experimental results reported in [31] show that this specimen experiences a delamination-dominated failure: the spread of transverse cracking in the plies, and the important amount of delamination associated lead to the coupon failure. Hence, the failure relies on the interaction between the transverse cracking in the plies and the delamination of the interface.

Concerning the damage evolution, the experiments show that the transverse cracking first develops in the upper $45^{\circ}$ ply, resulting in damage in the $45 / 90$ interface. Then, transverse cracking reaches the $90^{\circ}$ plies. Damage goes through plies and interfaces until the degradation of the $-45 / 0$ interface on the whole width of the coupon, which corresponds to the failure.

Because a large amount of subcritical damage occurs, the stress-strain curve experiences a slope change before the final breakdown.

In order to highlight the influence of the interface models on the damage evolution prediction, the test case is simulated using the enhanced interface model and a more classical one which does not include the intra-interlaminar coupling.

Remark Details concerning the material properties and finite element simulation features are presented in the paper [12].

\section{Simulation results: global behavior}

The stress-strain curves given by the two simulations are presented in Figure 7.

The two simulations show a slope change for a imposed strain $\varepsilon=0.38 \%$. This corresponds to the development of subcritical damage in the coupon which matchs the experimental observations.

The model including coupling predicts a failure stress close to the experimental one: $\sigma_{\max }=280 \mathrm{MPa}$ for the simulation versus $\sigma_{\max }=285 \mathrm{MPa}$ for the experimental value. The second one, that does not include coupling, predict a failure stress higher than the experimental one $\left(\sigma_{\max }=315 \mathrm{MPa}\right.$ vs $\left.\sigma_{\max }=285 \mathrm{MPa}\right)$.

In the following, the damage evolution predicted by the two models are compared. The study focuses on transverse cracking in the plies (represented in the damage charts by the

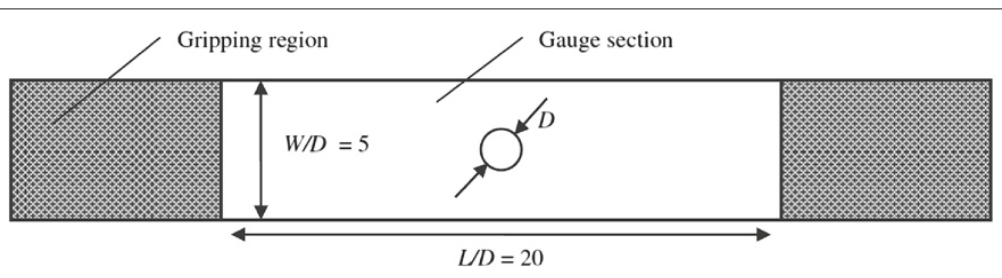

Figure 6 Geometry of the specimens. 


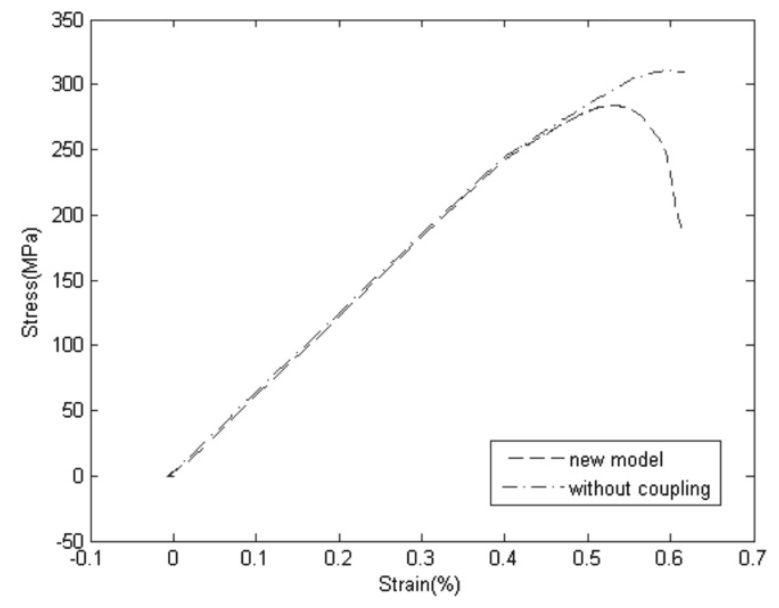

Figure 7 Stress-strain curves issued from the two simulations.

variable $\rho$ ) and delamination in the interfaces (represented by the variable $d_{I}$ ) as they are the main mechanisms concerned by the interface model.

The models are compared at four strain level:

1. $\varepsilon=0.38 \%$ : transverse cracking appears in the plies

2. $\varepsilon=0.42 \%$ : all plies experience transverse cracking

3. $\varepsilon=0.52 \%$ : transverse cracking has spread all over the width of the upper $45^{\circ}$ ply

4. $\varepsilon=0.58 \%$ : specimen has failed

\section{Damage prediction comparison: need of the intra-interlaminar coupling}

For $\varepsilon=0.38 \%$ (Figure 8 ), the two models give similar results in terms of transverse cracking. It appears in the upper ply and goes through plies and interfaces as described in [31].

For $\varepsilon=0.42 \%$ (Figure 9) and $\varepsilon=0.52 \%$ (Figure 10), the two models go on predicting similar behavior in terms of transverse cracking. However, whereas the model including coupling predicts a spread of delamination in the interfaces, the model without it does not predict any degradation of the interfaces.

For $\varepsilon=0.58 \%$ (Figure 11), the model including coupling leads to a delaminationdominated failure, as reported in [31], whereas the second model yields a fiber breaking dominated failure.

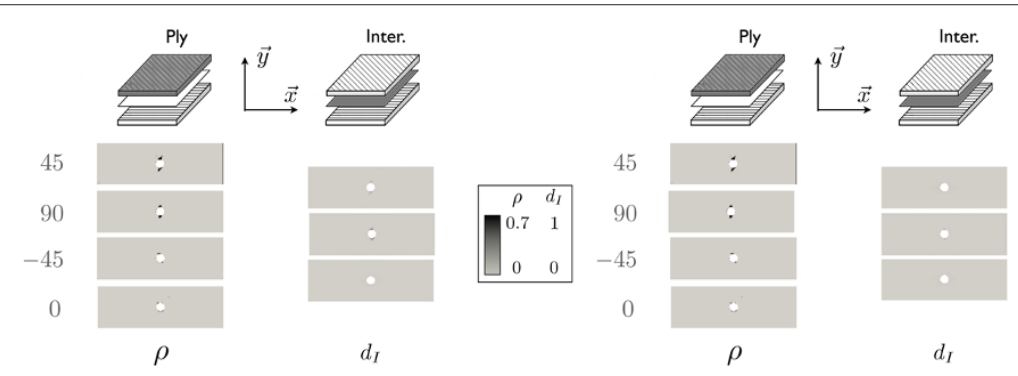

Figure 8 Damage charts yielded by models with (left) and without (right) coupling for a strain $\epsilon=0.38 \%$. 


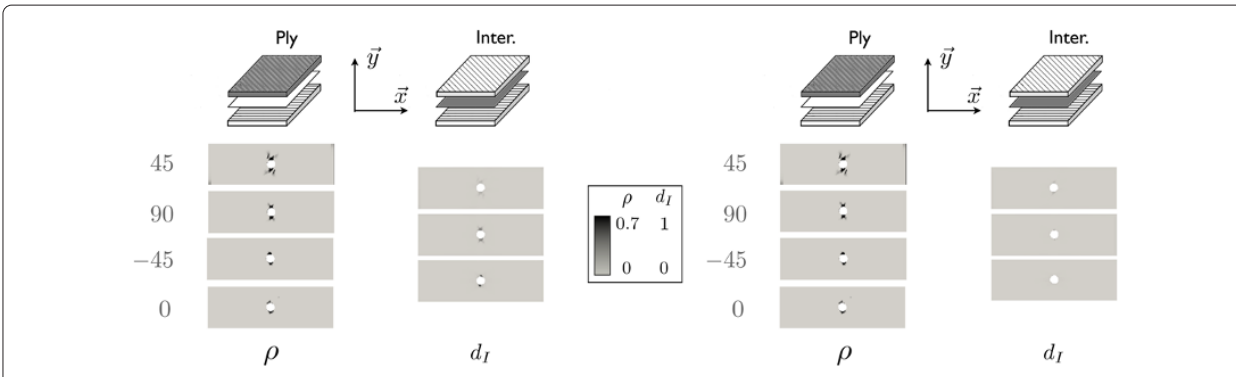

Figure 9 Damage charts yielded by models with (left) and without (right) coupling for a strain $\epsilon=0.42 \%$.

To resume, the two simulations predict similar behaviors for the transverse cracking, which match experimental observations. However, the enhanced model predicts a spread of delamination in the different interfaces almost as soon as transverse cracking appears, whereas the second model do not predict any delamination until an equivalent strain of $\varepsilon=0.5 \%$. This difference of behavior leads to different failure mode: the new model predicts a delamination dominated failure matching the experimental observations, the second model predicts a delayed failure due to fiber breaking.

These results highlight the need for introducing intra-/interlaminar's behavior coupling in order to accurately predict the damage evolution and failure stress and mode. More, the comparison with the experimental results illustrates the good capabilities of the enhanced interface model to predict the damage evolution and the failure pattern in the case of structural test cases such as open-hole tensile tests. Let us note that for this case the former version of our interface model gives similar results to the enhanced present one [12].

\section{Conclusion}

A new and relatively simple interface mesomodel taking into account the coupling with microcracking in the adjacent plies has been derived from the description of the damage scenarios on the microscale. This is a general model in which the damage states of the adjacent plies can be very different. Classical tests suffice to enable the identification of the material constants. The resulting enhanced mesomodel (ply and interface) is a computational model which is suitable for virtual testing. Indeed, it

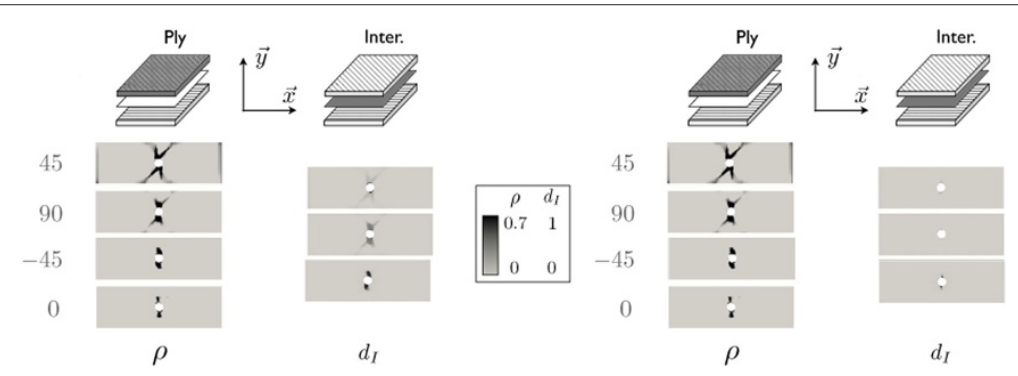

Figure 10 Damage charts yielded by models with (left) and without (right) coupling for a strain $\epsilon=0.52 \%$. 


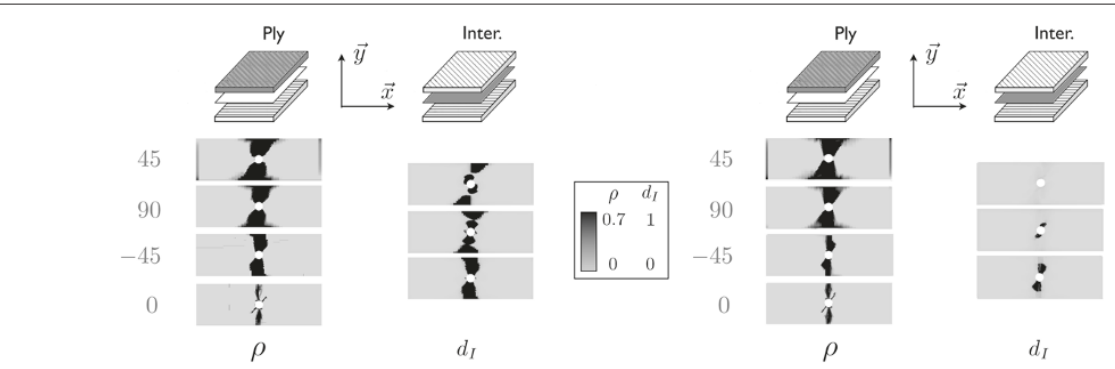

Figure 11 Damage charts yielded by models with (left) and without (right) coupling for a strain $\epsilon=0.58 \%$.

includes a physically sound description of situations involving intra/interlaminar coupling, thus it goes beyond the domain of validity of the standard mesomodel. Let us note also that the micro-meso bridge developed in this paper could be extended to the study of carbon/epoxy laminates interfaces interleaved with thermoplastic particles [32].

In this paper, the simulation of $\left[0 m / 90_{4}\right]_{s}$ and open-hole tensile tests showed that this model reproduces experimental observations quite well. A more complete validation, for plates with holes and low-velocity impact tests, even for ultra-thick laminates [33], will be addressed in companion papers. In these more complex cases, there remain some issues related to the numerical treatment of isolated transverse macrocracks, which tend to be wider in the simulations than in reality. This is a general question which is currently being addressed.

Moreover, computational cost being prohibitive for designers, another challenge has to be tackled using the laminates model presented here: the building of virtual charts i.e, reduced models including the description of uncertainties [34].

Competing interests

The authors declare that they have no competing interests.

\section{Authors' contributions}

All authors participated in the modeling and simulation work and in the redaction of the paper. All authors read and approved the final manuscript.

Received: 27 May 2013 Accepted: 24 September 2013

Published: 29 January 2014

References

1. Ladevèze P (2005a) Multiscale computational damage modelling of laminated composites, No. 474 in series, CISM Courses and Lectures. SpringerWien, New York

2. Herakovich CT (1998) Mechanics of fibrous composites. Wiley, New York

3. de Borst R, Schipperen JHA (2002) Continuum damage mechanics of materials and structures; chap. Computational Methods for delamination and fracture in composites. Elsevier. Amsterdam. pp 325-352

4. Ladevèze $P$, Lubineau G (2001) On a damage mesomodel for laminates: micro-meso relationships, possibilities and limits. Composite Sci Technol 61(15):2149-2158

5. Ladevèze P, Lubineau G (2002) An enhanced mesomodel for laminates based on micromechanics. Composite Sci Technol 62(4):533-541

6. Lopes CS, Camanho PP, Gürdal Z, Maimí P, Gonzáles EV (2009) Low-velocity impact damage on dispersed stacking sequence laminates. Part II: Numerical simulation. Composite Sci Technol 69(7-8):937-947

7. Flesher ND, Herakovich CT (2006) Predicting delamination in composite structures. Composite Sci Technol 66(6):745-754

8. Greve L, Pickett AK (2006) Delamination testing and modelling for composite crash simulation. Composite Sci Technol 66(6):816-826

9. Johnson AF, Holzapfel M (2003) Modelling soft body impact on composite structures. Composite Struct 61(1-2):103-113

10. Guinard S, Allix O, Guédra-Degeorges D, Vinet A (2002) A 3D damage analysis of low-velocity impacts on laminated composites. Composite Sci Technol 62(4):585-589 
11. Daghia F, Ladevèze $P$ (2013) Identification and validation of an enhanced mesomodel for laminated composites within the WWFE-III. J Composite Mater 47(20-21): 2675-2693

12. Abisset $E$, Daghia $F$, Ladevèze $P$ (2011) On the validation of a damage mesomodel for laminated composites by means of open-hole tensile tests on quasi-isotropic laminates. Composite Part A 42:1515-1524

13. Harris CE, Morris DH (1985) Delamination and Debonding of Materials; chap In: Role of delamination and damage development on the strength of thick notched laminates STP 876. ASTM, Philadelphia, pp 424-447

14. Nairn JA, Hu S (1992) The initiation and growth of delaminations induced by matrix microcracks in laminated composites. Int J Fracture 57(1):1-24

15. Finn SR, He YF, Springer GS (1993) Delaminations in composite plates under transverse impact loads - experimental results. Composite Structures 23(3):191-204

16. Eggers H, Goetting HC, Bäuml H (1994) Synergism between layer cracking and delaminations in multidirectional laminates of carbon-fibre-reinforced epoxy. Composite Sci Technol 50(3):343-354

17. Chen WH, Yang SH (1996) Multilayer hybrid-stress finite element analysis of composite laminates with delamination cracks originating from transverse cracking. Eng Fracture Mech 54(5):713-729

18. Johnson P, Chang FK (2001 b) Characterisation of matrix crack-induced laminate failure - Part II: Analysis and verifications. J Composite Mater 35(22):2037-2074

19. Li S, Reid SR, Zou Z (2006) Modelling damage of multiple delaminations and transverse matrix cracking in laminated composites due to low velocity lateral impact. Composite Sci Technol 66(6):827-836

20. Zhang $H$, Minnetyan $L$ (2006) Variational analysis of transverse cracking and local delamination in $\left[\theta_{m} / 90_{n}\right]_{s}$ laminates. Int J Solid Struct 43:7061-7081

21. Nairn JA, Hu S (1994) Damage Mechanics of Composite Materials; chap. Matrix Microcracking. Elsevier Science, pp 187-243

22. Nairn JA (2000) Comprehensive Composite Materials: Polymer Matrix Composites; chap. Matrix Microcracking in Composites. Pergamon Press, Oxford

23. Ladevèze $P$ (2005b) Mechanics of the 21st Century; chap. A bridge between the micro- and mesomechanics of laminates fantasy or reality? Springer, Dordrecht, pp 187-201

24. Violeau D, Ladevèze P, Lubineau G (2009) Micromodel-based simulations for laminated composites. Composite Sci Technol 69(9):1364-1371

25. Ladevèze P, Lubineau G, Violeau D (2006a) A computational damage micromodel of laminated composites. Int J Fracture 137(1-4):139-150

26. Hallett SR, Jiang WG, Khan B, Wisnom MR (2008) Modelling the interaction between matrix cracks and delamination damage in scaled quasi-isotropic specimens. Composite Sci Technol 68(1):80-89

27. Bouvet C, Castanié B, Bizeul M, Barrau J-J (2009) Low velocity impact modelling in laminate composite panels with discrete interface elements. Int J Solids Struct 46(14-15):2809-2821

28. Van der Meer FP, Sluys $\sqcup J$ (2010) Mesh-independent modeling of both distributed and discrete matrix cracking in interaction with delamination in composites. Eng Fracture Mech 77(4):719-735

29. Ladevèze P, Lubineau G, Marsal D (2006b) Towards a bridge between the micro- and mesomechanics of delamination for laminated composites. Composite Sci Technol 66:698-712

30. Hashin Z (1996) Finite thermoelastic fracture criterion with application to laminate cracking analysis. J Mech Phys Solids 7:1129-1145

31. Wisnom MR, Hallett SR (2009) The role of delamination in strength, failure mechanism and hole size effect in open hole tensile tests on quasi-isotropic laminates. Composites 40(1):335-342

32. Gao F, Jiao G, Lu Z, Ning R (2007) Mode II delamination and damage resistance of carbon/epoxy laminates interleaved with thermoplastic particles. J Composite Mater 41:111-123

33. Czichon S, Zimmermann K, Middendorf P, Vogler M, Rolfes R (2011) Three-dimensional stress and progressive failure analysis of ultra thick laminates and experimental validation. Composite Structures 93:1394-1403

34. Chinesta F, Ladeveze P, Cueto E (2011) A short review on model order reduction based on Proper Generalized Decomposition. Arch Comput Methods Eng 18:395-404

doi:10.1186/2213-7467-1-7

Cite this article as: Ladevèze et al:: A micromechanics-based interface mesomodel for virtual testing of laminated composites. Advanced Modeling and Simulation in Engineering Sciences 2013 1:7.

\section{Submit your manuscript to a SpringerOpen ${ }^{\circ}$ journal and benefit from:}

- Convenient online submission

$\rightarrow$ Rigorous peer review

- Immediate publication on acceptance

- Open access: articles freely available online

- High visibility within the field

- Retaining the copyright to your article

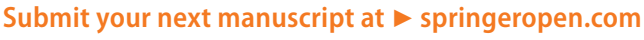

